\title{
EL DESEMPLEO JUVENIL EN LATINOAMÉRICA Y EL EMPRENDIMIENTO DE ESTUDIANTES UNIVERSITARIOS ${ }^{1}$
}

\author{
YOUTH UNEMPLOYMENT IN LATIN AMERICA AND THE ENTREPRENEURSHIP
} OF UNIVERSITY STUDENTS

\section{DESEMPREGO JUVENIL NA AMÉRICA LATINA E O EMPREENDEDORISMO DE ESTUDANTES UNIVERSITÁRIOS}

\author{
SAAVEDRA GARCÍA_ María Luisa
}

Doctora en Administración, Universidad Nacional Autónoma de México. Profesora titular, Facultad de Contaduría y Administración, Universidad Nacional Autónoma de México. E-mail: lsaavedra@fca.unam.mx, México.

Recibido: 23 de julio de 2020

Aprobado: 31 de julio de 2020

DOI: https://doi.org/10.22267/rtend.202102.151

\section{RESUMEN}

Este artículo tiene como objetivo, brindar un panorama del desempleo juvenil en Latinoamérica y analizar de qué manera el emprendimiento podría ser la solución a este problema. Se realizó una investigación documental, revisando informes elaborados por organismos internacionales, y trabajos científicos realizados sobre el tema. Los principales hallazgos muestran que el desempleo en esta región afecta mayormente a los jóvenes, por lo que es necesario reforzar la situación educativa de los jóvenes; se encontró también, que los jóvenes universitarios están motivados para emprender principalmente por el deseo de poner en práctica una idea innovadora y por haber crecido en un entorno familiar empresarial, sin embargo, a pesar de los esfuerzos de los programas de fomento internacionales, existen aún muchas barreras que dificultan el emprendimiento juvenil, en esta región.

\footnotetext{
${ }^{1}$ Se agradece el apoyo del Programa de Apoyo a Proyectos para la Innovación y Mejoramiento de la Enseñanza (PAPIME), para el desarrollo del proyecto PE300120 "Material didáctico para fortalecer la intención de emprendimiento en estudiantes universitarios y propuesta de asignaturas para proporcionar competencias empresariales".
} 
Palabras clave: desempleo; emprendimiento; estudiantes universitarios; jóvenes; trabajo. JEL: E24; J23; J64; J71; L26

\begin{abstract}
This article aims to provide an overview of youth unemployment in Latin America and analyze how entrepreneurship could be the solution to this problem. A documentary investigation was carried out, reviewing reports prepared by international organizations, and scientific works carried out on the subject. The main findings show that unemployment in this region affects mainly young people, so it is necessary to reinforce the educational situation of young people; It was also found that young university students are motivated to undertake mainly by the desire to implement an innovative idea and by having grown up in a family business environment, however, despite the efforts of international development programs, there are still many barriers that hinder youth entrepreneurship in this region.
\end{abstract}

Keywords: unemployment; entrepreneurship; university students; young people; work.

JEL: E24; J23; J64; J71; L26

\title{
RESUMO
}

Este artigo tem como objetivo fornecer uma visão geral do desemprego jovem na América Latina e analisar como o empreendedorismo pode ser a solução para esse problema. Foi realizada uma investigação documental, revisando relatórios elaborados por organizações internacionais e trabalhos científicos realizados sobre o assunto. Os principais resultados mostram que o desemprego nessa região afeta principalmente os jovens, sendo necessário reforçar a situação educacional dos jovens; Constatou-se também que jovens estudantes universitários são motivados a empreender principalmente pelo desejo de implementar uma idéia inovadora e por terem crescido em um ambiente de negócios familiares, no entanto, apesar dos esforços dos programas de desenvolvimento internacional, ainda existem muitas barreiras que impedem o empreendedorismo juvenil nessa região.

Palavras-chave: desemprego; empreendedorismo; estudantes universitários; jovens; trabalham. JEL: E24; J23; J64; J71; L26 


\section{INTRODUCCIÓN}

El desempleo es un problema que aqueja a los países en Latinoamérica en un contexto de desaceleración económica, donde uno de cada cinco jóvenes se encuentra desempleado y esta tasa es cinco veces superior al desempleo en general; esta situación trae como consecuencia la falta de oportunidades de trabajo decente para los jóvenes de la región lo cual es motivo de preocupación, dado que causa desaliento y frustración en este sector de la población haciéndolo vulnerable, orillándolos a aceptar trabajos informales donde se desempeña el $60 \%$ de la población juvenil, este tipo de trabajos por lo general son precarios, es decir con bajos salarios y sin prestaciones laborales, agudizando así el círculo de pobreza en estos jóvenes (OIT, 2019).

El problema se acentúa más aún si se considera que de los jóvenes entre 15-29 años el 60\% no estudian, siendo superior esta proporción en México y Centroamérica (OIT, 2019), esto implica que la gran desigualdad que impera en la región orilla a los jóvenes a buscar un empleo para subsistir o para apoyar la economía familiar, dejándolos así rezagados de una educación técnica o profesional que les proporcionaría la oportunidad de obtener empleos dignos y mejor remunerados. Esta realidad latente se presenta como un desafío para los gobiernos e instituciones que deben generar alternativas de solución viables para que los jóvenes mejoren sus expectativas de vida, es aquí entonces donde aparece el emprendimiento juvenil, el cual ofrece una alternativa de autoempleo para los jóvenes de la región.

Naciones Unidas (2020) refiere que el emprendimiento y el autoempleo brindan oportunidades económicas para que una amplia población mundial conformada por hombres y mujeres jóvenes, genera empleos en el sector privado; la mayoría de los 1.200 millones de jóvenes del mundo viven en economías en desarrollo, donde las pequeñas y medianas empresas (PYME) representan el 52\% del empleo total, estas y las empresas jóvenes suelen ser más dinámicas que las grandes empresas en lo que respecta al crecimiento del empleo. Sin embargo, con un número creciente de jóvenes que ingresan al mercado laboral y oportunidades limitadas para la creación de empleo, el desempleo y la desvinculación amenazan el desarrollo sostenible y la estabilidad social y ocasiona que las personas migren en busca de trabajo. Cuando los jóvenes adquieren las habilidades y oportunidades económicas para desarrollar su potencial, este dividendo demográfico puede ayudar a estimular la creación de empleo dirigida a los mismos jóvenes. 
De aquí la necesidad de realizar un proyecto académico, que busca impulsar la intención de emprendimiento en estudiantes universitarios a través de elaboración de material didáctico y propuesta de asignaturas, para lo que en primer lugar se necesita conocer la situación actual de los jóvenes, así como las motivaciones que tienen los universitarios para iniciar emprendimientos.

Por lo anterior la pregunta de investigación es ¿Cuál es la situación de desempleo en los jóvenes y, puede el emprendimiento ser una solución a la situación de los jóvenes Latinoamericanos? Así pues, este artículo tiene como objetivo, brindar un panorama del desempleo juvenil en México y Latinoamérica y analizar de qué manera el emprendimiento podría ser la solución a este problema. Por lo cual en primer lugar se describe el problema del desempleo juvenil en Latinoamérica, posteriormente, se describe el emprendimiento juvenil y las motivaciones de los jóvenes universitarios para emprender y, por último, se presentan la discusión y conclusiones de este trabajo.

\section{REFERENTES TEÓRICOS}

\subsection{El problema del desempleo juvenil en Latinoamérica}

De acuerdo con OECD/ECLAC/CAF (2016), los jóvenes con edades de 15 a 29 años en América Latina y el Caribe (ALC) son más 163 millones, ósea la cuarta parte de la población de esta región. Por su parte, Worldwide (2014) manifiesta que de los 7 mil millones de habitantes en el mundo la mitad son personas menores de 27 años de edad, en Latinoamérica viven 110 millones (OIT, 2019) lo cual representa un gran desafío ante un panorama de desaceleración económica, pues se presenta el riesgo de no alcanzar el progreso social, político y económico suficiente para que la población pueda contar con una vida digna, en medio de este escenario y riesgo se encuentran los jóvenes como actores centrales, pues se estima que en el mundo existen 73,4 millones de jóvenes desempleados (Worldwide, 2014).

Si bien es cierto, el progreso social y económico de las últimas décadas, ocasionaron que los jóvenes se beneficiaran de la bonanza económica y el progreso social, la cual logró abatir la pobreza y reducir la desigualdad; se necesita avanzar más, pues la realidad indica que de los jóvenes que trabajan el $60 \%$ lo hace en el sector informal y un $22 \%$ no realiza ninguna actividad (OIT, 2019). Este contexto provocó que se implementaran políticas públicas más incluyentes y con la participación de los sectores sociales que habían estado históricamente relegados, por consecuencia la clase media consolidada logró avanzar 14 puntos porcentuales en el último 
decenio de años, alcanzado a más del 35\% de la población Latinoamericana. A pesar de esto, una proporción importante de latinoamericanos jóvenes viven en hogares pobres o vulnerables (64\% de los latinoamericanos jóvenes es decir 110 millones, en comparación con 57\% de los adultos), donde la mayoría de ellos soportan servicios de mala calidad, empleos precarios e informales, escasos ahorros y poca movilidad social (OECD/ECLAC/CAF, 2016; ILO, 2018, OIT, 2019).

Esto como consecuencia de que jóvenes en una alta proporción abandonan sus actividades escolares sumándose al desempleo o a empleos informales y precarios en América Latina y el Caribe (OECD/ECLAC/CAF, 2016); así pues, el 47\% de estos jóvenes se encuentran laborando en la informalidad, siendo preocupante el aumento de esta tendencia puesto que al año 2018, este porcentaje alcanzó el 62.6\% del total de los jóvenes ocupados (ILO, 2018); siendo más recurrente esta situación informalidad entre los jóvenes de hogares pobres y vulnerables, comparados con los de clase media. Por otra parte, el 20\% de los jóvenes (22 millones) de Latinoamérica se encuentra desempleado, no cursa estudios, ni capacitación para el empleo, por lo que quedan fuera de los dos vías principales de inclusión social y económica: el sistema educativo y el mercado laboral; esta situación se aprecia mayormente entre los jóvenes de Honduras, El Salvador, Guatemala y México, donde alcanzan más del $25 \%$, donde los jóvenes de estratos socioeconómicos más bajos son los que se encuentran en esta situación.

Resulta preocupante conocer que la tasa de desempleo juvenil en esta región es el doble de la tasa mundial general y el triple de los adultos de la región y que además uno de cada cinco jóvenes que busca trabajo no lo encuentra y si lo encuentra se trata de un trabajo precario (OIT, 2019). Aunado a esto, los jóvenes son lo que resultan más perjudicados cuando se presentan crisis económicas, puesto que son más propensos a ocuparse en empleos temporales (OECD/ECLAC/CAF, 2016), a este respecto Worldwide (2014) da cuenta de que en los países en desarrollo seis de cada diez trabajadores de entre 15-29 años carecen de contrato de trabajo, reciben salarios por debajo del promedio y ocho de cada diez están en el sector informal. Traduciéndose esto, que se encuentran en un sector de trabajos precarios, baja productividad, bajos ingresos y pocas oportunidades para elevar su nivel de vida (Worldwide, 2014), pues los salarios que reciben se ubican en el umbral de la pobreza US \$ 1.90 por día (ILO, 2017).

Así pues, las condiciones laborales de los jóvenes reflejan la precariedad de los empleos a los que están accediendo dada la falta de oportunidades en la región para obtener mejores empleos. En 
referencia al tipo de contrato la Figura 1, muestra una información más actualizada donde en la gran mayoría de los países de ALC, más de la mitad de los jóvenes ocupados se encuentran contratados ya sea de manera temporal o sin un contrato escrito (salvo en Argentina, Costa Rica y Chile). En Perú, Paraguay y México predomina la contratación temporal, aunque si bien es cierto los contratos temporales, ya sea de prueba o de tipo formativo (e. g. programas de aprendices, formación dual, etc.), son deseables en la medida que facilitan el proceso de adaptación entre empleador y empleado. Sin embargo, aquellos jóvenes que son empleados como un mecanismo para reducir los costos de despido podrían tener consecuencias adversas en el empleo y la productividad; más aún, cuando algunos estudios muestran una asociación entre relaciones laborales de corta duración (como es el caso de los contratos temporales) con una menor incidencia en capacitación, donde una menor inversión en formación podría tener repercusiones en términos de productividad (OIT, 2019).

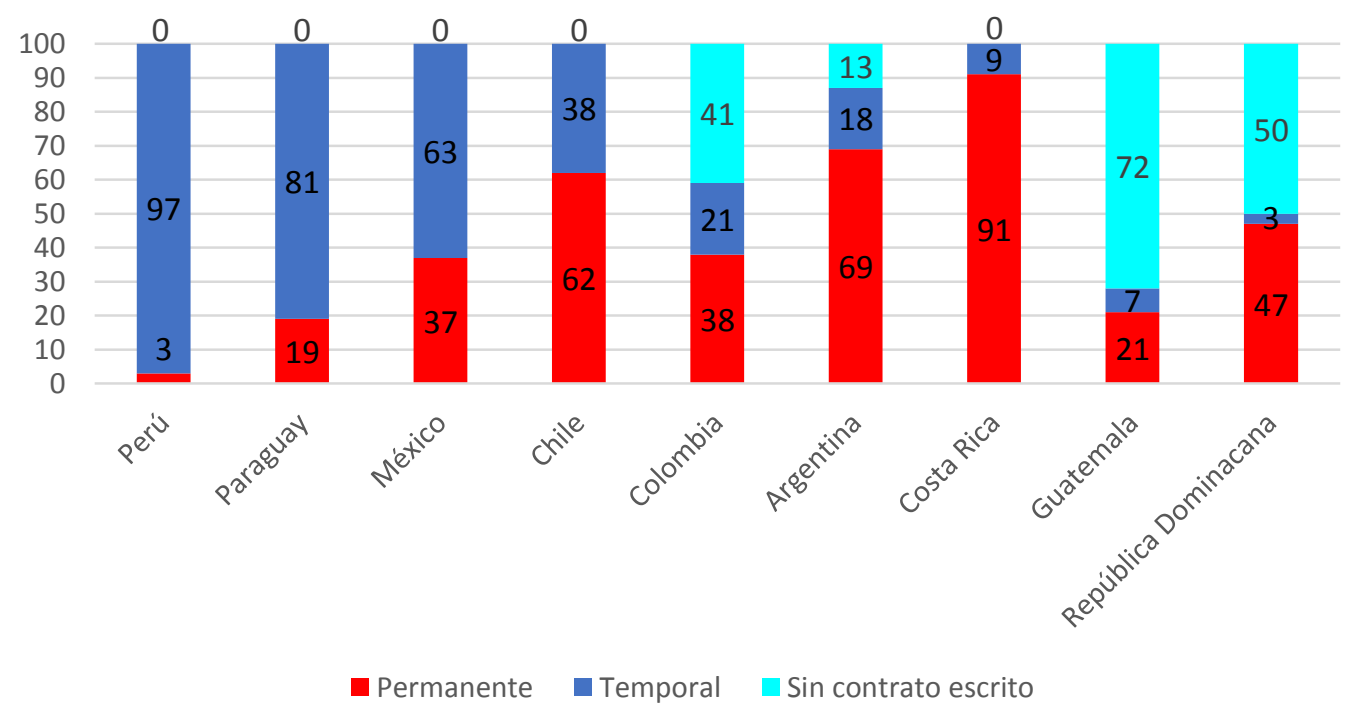

Figura 1. Jóvenes (15 a 29 años) ocupados según modalidad de contrato en países seleccionados de América Latina y el Caribe. 2018 (Porcentajes)

Fuente: OIT (2019).

Esta situación, implica que una carga desproporcionada de desempleo recae sobre los jóvenes, tal como se aprecia en la Figura 2, donde se muestra claramente como los hombres y mujeres adultos tienen mayores tasas de ocupación que los hombres y mujeres jóvenes (15 a 29 años). Esta tendencia prevalece pues ILO (2018) da cuenta que esta tasa a 2018 fue 19.6\%, mientras que el desempleo de la población adulta alcanzó el 6.3\%; así pues, de cada 100 jóvenes que buscan 
empleo 20 no lo consiguen, poniendo así en riesgo su desarrollo personal, económico y social, así como su futuro inmediato.

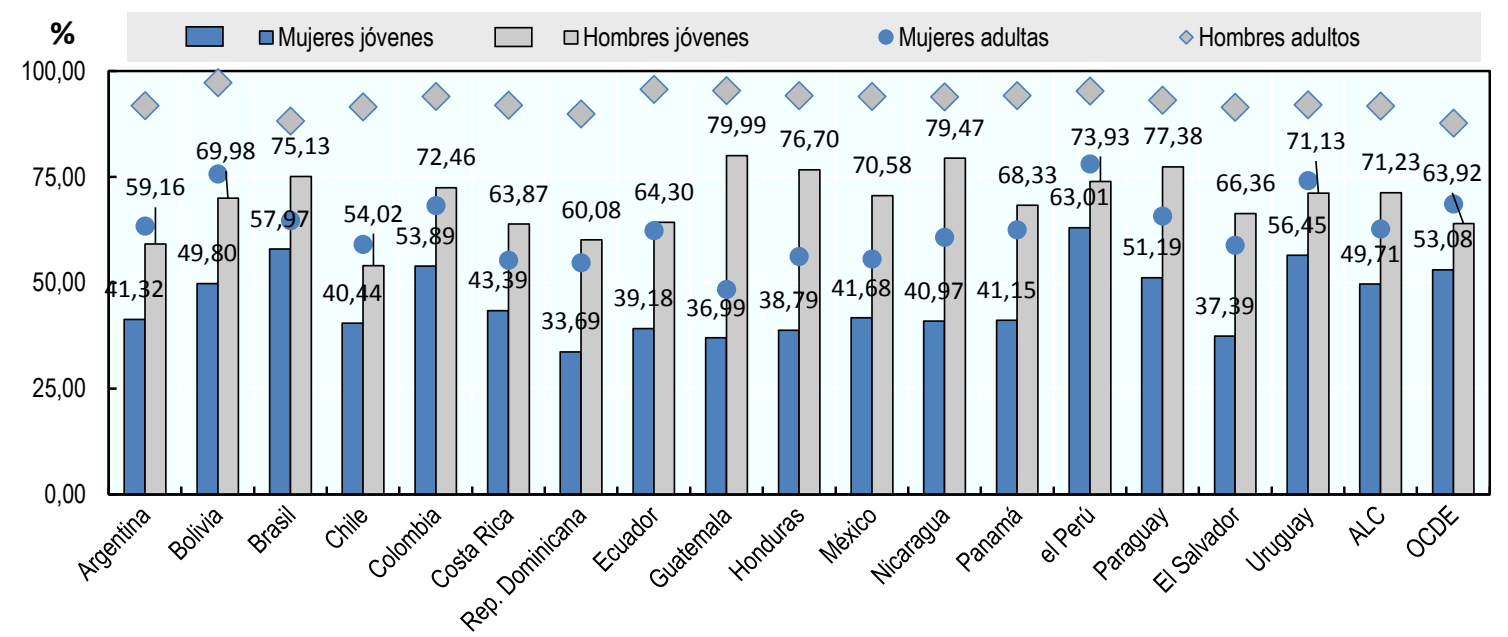

Figura 2. Tasa de participación en la fuerza de trabajo.

Fuente: OECD/ECLAC/CAF (2016).

Por otra parte, la tasa de desempleo de los jóvenes de niveles socioeconómicos muy bajos es decir jóvenes de hogares considerablemente pobres es del 24,6\%, el $20 \%$ para los de hogares moderadamente pobres y el 13,4\% para los de hogares vulnerables, en cambio los jóvenes de clase media sólo alcanzan 7\%. Las tasas de desempleo más elevadas se dan entre jóvenes de 15 a 29 , con un máximo de $28 \%$ para los que están en situación de pobreza extrema, el $25 \%$ para los que están en pobreza moderada y el 19\% para los que son vulnerables, frente al 12\% de los jóvenes en situación no vulnerable. Así también, a menor edad mayor tasa de desempleo tal como se muestra en la Figura 3, en lo que se refiere a las zonas geográficas, la tasa de desempleo es más elevada en zonas urbanas que en zonas rurales y castiga más a las mujeres jóvenes que a los hombres debido a los estereotipos de género que de la sociedad se permean a las organizaciones, constituyéndose en un obstáculo para que las mujeres participen en el mercado laboral (García, 2015; OECD/ECLAC/CAF, 2016; OIT, 2019). 


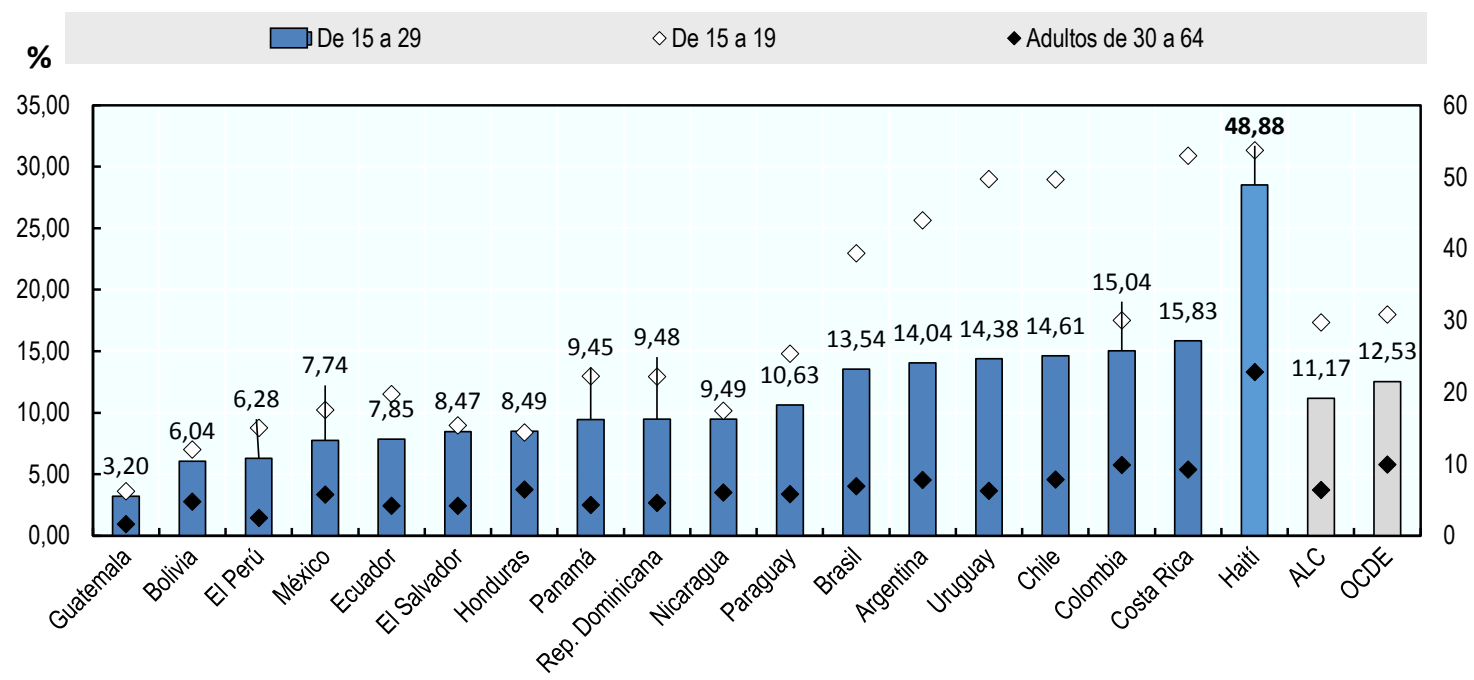

Figura 3. Tasa de desempleo por países, 2016.

Fuente: OECD/ECLAC/CAF (2016), OIT (2019).

La tendencia del desempleo juvenil va en aumento como se aprecia en la Figura 4 donde el país con un porcentaje más elevado es Brasil con $29 \%$ en 2018, mientras que Guatemala es el de menor proporción con un porcentaje de 6.1\% (ILO, 2018). Esta situación representa un foco de atención en la región pues las altas tasas de desempleo en los jóvenes impiden la obtención de oportunidades de empleo, educación y movilidad social.

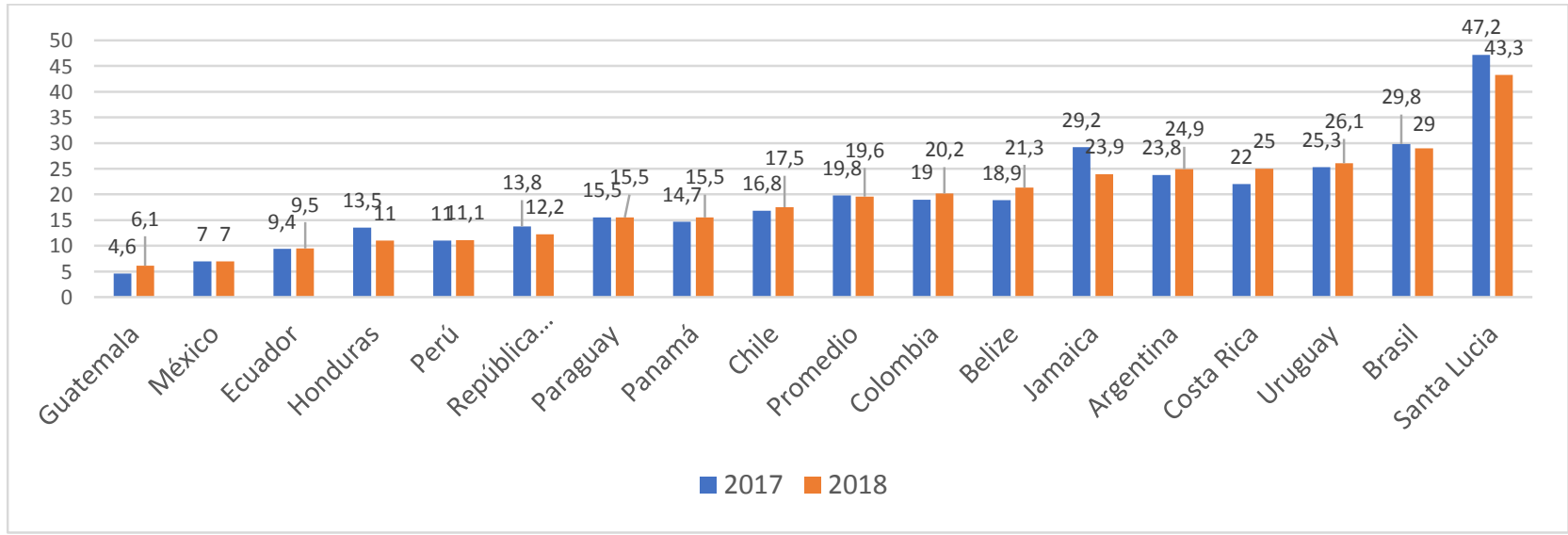

Figura 4. Tasas de desempleo Juvenil en Latinoamérica, 2017-2018 (Porcentaje)

Fuente: ILO (2018).

En cuanto a tipo de relación laboral más generalizado entre los jóvenes de 15 a 24 años se encuentra en primer lugar el trabajo asalariado: en promedio, 7 de cada 10 jóvenes trabajan en relación de dependencia, esta proporción que se incrementó entre 2010 y 2018 en alrededor de un punto porcentual, pasando de 70,6\% a 71,4\%. En un segundo lugar está el trabajo independiente el cual 
se mantuvo cercano al $14 \%$ durante mismo período. El trabajo familiar no remunerado (TFNR) que ocupa también, una parte importante del empleo juvenil (pasó del 14,3\% en 2010 al 3,1\% en 2018). Por último, el porcentaje de jóvenes dueños de empresas con trabajadores a su cargo no llega ni al 1\%, y se mantuvo sin cambio dentro del mismo período. En contraparte de esto se observa, que para los adultos la porción de empleo asalariado gira en torno al 60\%, mientras el porcentaje de trabajadores adultos independientes y patrones alcanza el 32\%, muy por encima de la tasa de los jóvenes, el empleo no remunerado representa menos del $4 \%$ para los adultos (ver Figura 5).

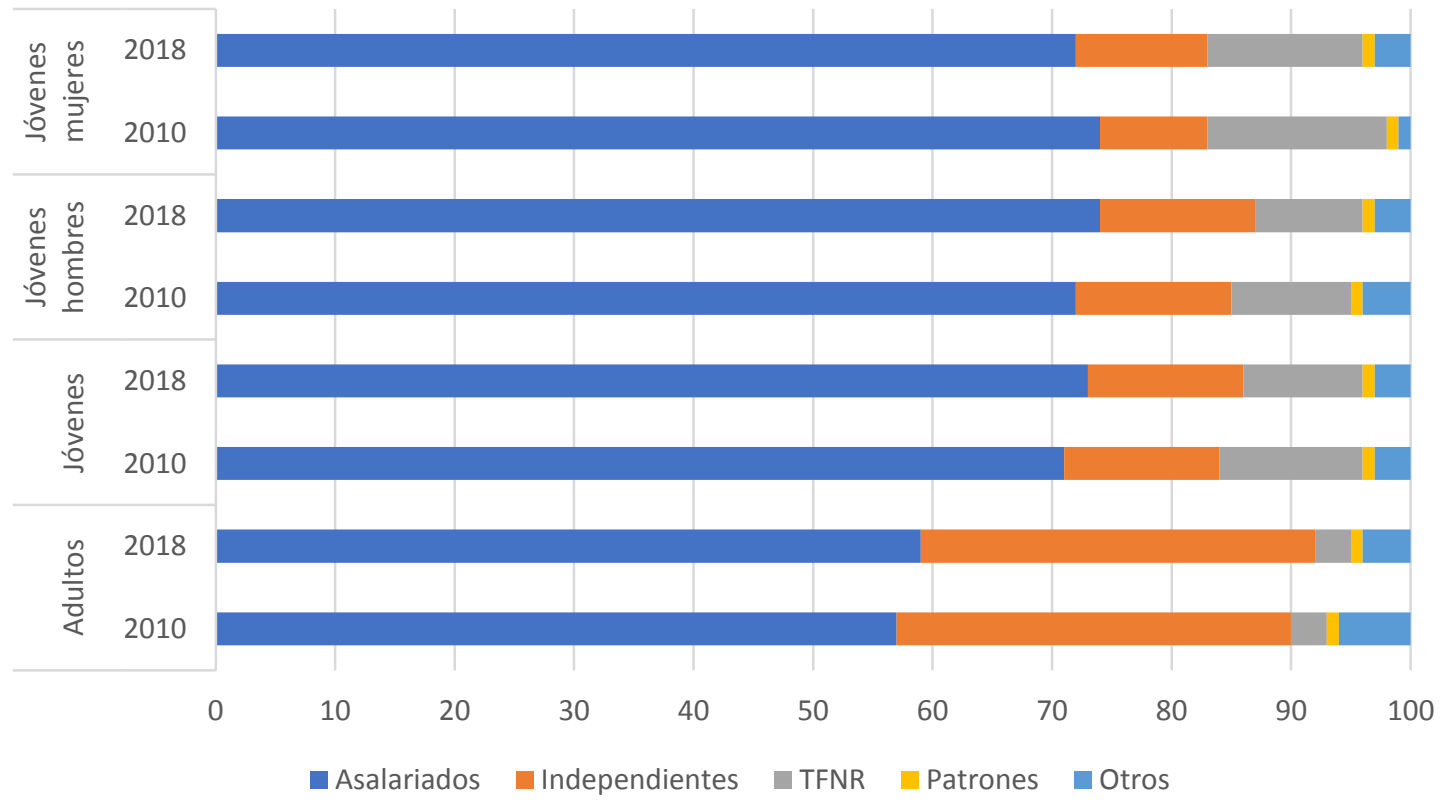

Figura 5. América Latina (17 países): categoría ocupacional de los jóvenes (15 a 24 años) y adultos (+25 años) - en porcentaje, 2010 y 2018.

Fuente: OIT (2019).

En lo que se refiere a las ramas de actividad, los jóvenes se encuentran principalmente empleados en los sectores de comercio, servicios no mercantiles (administración pública y otras actividades de servicios comunitarios y sociales) y manufactura. En su conjunto, para 2018, estos tres sectores representaban más del 50\% del empleo juvenil en los cinco países de América Latina y el Caribe considerados (con excepción de Perú). Por su parte, las mujeres jóvenes tienden a concentrarse en el sector de servicios no mercantiles vinculado al cuidado (educación, salud, actividades del hogar), siendo esta participación más del doble de la de los hombres; también es mayoritaria esta participación, en los sectores de comercio y hotelería o alimentación, salvo en el caso de República Dominicana. Mientras que en los sectores de agricultura, construcción, transporte, 
almacenamiento y comunicación se encuentran ocupados más del 30\% de los hombres jóvenes y menos del $13 \%$ de las mujeres (excepto en Perú) (OIT, 2019).

\subsection{El emprendimiento juvenil en Latinoamérica}

En este contexto el emprendimiento es particularmente importante como solución para mejorar el las condiciones de empleo y la calidad de vida entre los jóvenes, además, Worldwide (2014) refiere que se debe aprovechar la energía, el talento y la creatividad que estos tienen, por lo que es necesario desarrollar estrategias que impulsen esta actividad, puesto que es fundamental para mejorar las oportunidades y condiciones de vida de estos jóvenes (OECD/ECLAC/CAF, 2016; OECD, 2020). Contemplando que en Latinoamérica habitan más de 163 millones de personas de entre 15 y 29 años, de las cuales solo la mitad tiene empleo, se enfrenta al desafío de crear oportunidades laborales para los jóvenes especialmente en el sector formal.

Considerando lo anterior, CAF (2013) refiere que en Latinoamérica los jóvenes que se inician en un emprendimiento, son en mayor proporción los que se encuentran desempleados, en comparación de lo que ocurre en los países desarrollados; estos principalmente se convierten en trabajadores por cuenta propia es decir no contratan empleados, lo cual trae como consecuencia empresas de baja productividad con muy poca probabilidad de escalar a empresa grande, y con mayor probabilidad de fracasar, dado que se crean como una solución problema del desempleo. Coincidiendo con los resultados de GEM (2015) donde el 78\% de los emprendedores jóvenes en esta región inician sus empresas sin empleados, el 18\% tienen de 1 a 4 personas, el $4 \%$ de 4 a 19 personas y sólo el $1 \%$ con más de 20 personas.

Así pues, un gran número de jóvenes emprendedores Latinoamericanos lo son por cuenta propia, son dueños de microempresas, con pocos empleados y generan menos productividad, lo que ocasiona una propensión mayor que los países de OCDE a convertirse en emprendedores de subsistencia (OECD/ECLAC /CAF, 2016). Por otra parte, GEM (2015) refiere que el 52\% de los jóvenes emprendedores de esta región emprenden con ahorros personales, el 16\% con ahorros de la familia, el $26 \%$ con préstamos bancarios o de otras instituciones financieras y el $2 \%$ con préstamos de amigos.

Por medio del emprendimiento, los jóvenes pueden incorporarse a los mercados laborales y generar beneficios positivos en el mediano plazo, tales como la introducción de productos 
innovadores, creación de empleos, mejorar el capital humano, entre otros, es importante mencionar que el emprendimiento es una estrategia para facilitar la participación económica de los jóvenes (Appie, Eresia y Gervase, 2014). Así también, los jóvenes al desarrollar esta actividad pueden adquirir nuevas competencias y mejorar su bienestar, contrarrestando así los efectos perjudiciales del desempleo, que lo conducen a un círculo vicioso de bajos salarios y desempleo prolongado, además, los jóvenes emprendedores de la región con sus logros pueden llegar a ser modelos para otros jóvenes al demostrar su potencial emprendedor (OECD/ECLAC/CAF, 2016).

Los jóvenes emprendedores de ALC, presentan desigualdades importantes por un lado se muestra la existencia de pocos emprendedores de alto crecimiento y por el otro, muchos emprendedores de subsistencia; esto a pesar de contar con las mismas motivaciones y actitudes hacia el emprendimiento que los jóvenes de los países de la OCDE, puesto que la iniciativa de los jóvenes hacia el emprendimiento en ALC tiene alta dependencia con la estructura del mercado laboral y la composición empresarial de la región. Así pues, los jóvenes emprendedores de esta región generalmente son trabajadores por cuenta propia, de estratos socioeconómico más bajos, y con niveles inferiores de educación, como consecuencia la proporción de emprendedores de subsistencia entre los jóvenes de Latinoamérica es amplia.

La región Latinoamericana se caracteriza por el predominio de micro, pequeñas y medianas empresas que representan en promedio el $90 \%$ del total del tejido empresarial y generan en promedio el $80 \%$ del empleo; siendo que las empresas grandes son menores en cantidad, pero contribuyen con el 70\% del Producto Interno Bruto, mostrando así una gran asimetría entre empleo y productividad, situación que se replica en los emprendimientos (OECD/ECLAC/CAF, 2016, OECD, 2019). Así también, Acosta, Zárate e Ibarra (2014), señalan que los países de Latinoamérica tienen bajo desarrollo económico pero altos niveles de emprendimiento y que este se da principalmente por necesidad, lo que implica que es muy baja la motivación para emprender por oportunidad.

Es así entonces como en esta región coexisten los emprendedores de alto crecimiento y los emprendedores de subsistencia, asociándose esta actividad con el microemprendimiento informal donde no se ha encontrado todavía como salvar las barreras que impiden el desarrollo de emprendedores productivos, replicándose esta situación en los emprendedores jóvenes; los cuales son trabajadores por cuenta propia y tienden a poseer empresas de subsistencia, los cuales 
pertenecen a los estratos económicos más bajos. Así, pues estos emprendedores procedentes de hogares de ingresos medios y bajos, afrontan más barreras para emprender pues cuentan con menos recursos, competencias y redes, que los que provienen de hogares de ingresos altos, los cuales son más propensos a una mayor exposición de experiencia empresarial, familiar y escolar desde temprana edad en comparación con los de ingresos medios y bajos (OECD/ECLAC/CAF, 2016). Por su parte, Hulsink y Koek (2014) refieren que los emprendedores jóvenes no se preocupan por la preparación universitaria y aprenden sobre empresas ya sea en casa con sus padres, amigos o compañeros, aunque en un número limitado de casos son descendientes de padres emprendedores.

Esta sería la razón por lo que la educación que poseen los jóvenes emprendedores Latinoamericanos es menor en promedio que la de los jóvenes de los países de la OCDE, sin embargo, los emprendedores jóvenes en esta región cuentan con niveles de estudios más elevados que los emprendedores adultos; y aunque la proporción de emprendedores jóvenes es similar a los de homólogos de la OCDE en educación secundaria, en los que se refiere a la educación terciaria la brecha es amplia al alcanzar únicamente el 13\% vs. un 33\% de la OCDE (OECD/ECLAC /CAF, 2016). Por otra parte, GEM (2015) refiere que el $20 \%$ de los jóvenes emprendedores en esta región, manifiestan haber recibido entrenamiento para el emprendimiento en la escuela, mientras que el $21 \%$ lo ha recibido fuera de la escuela y el $70 \%$ no ha recibido ningún entrenamiento para emprender.

En cuanto a las actitudes hacia el emprendimiento, son similares tanto en los países de Latinoamérica como en los de la OCDE; en ambas regiones se considera a la actividad emprendedora como potenciadora del status social, así pues, la elección del empleo es afectada por la percepción de los pares. En Latinoamérica el status de empresario se asocia con trabajo duro, posibles ingresos elevados y bajo nivel educativo, se encuentra muy asociada la idea de emprendedor exitoso y elevado status social, calidad de vida y el poder usar su creatividad, por lo que se percibe positivamente la actividad de emprendimiento, visualizando que le otorgará poder, seguridad y dinero, así como el poder hacer algo útil por su comunidad (OECD/ECLAC/CAF, 2016).

Sin embargo, OECD/ECLAC, CAF (2016) dan cuenta que en Latinoamérica es más elevado el emprendimiento por necesidad y se encuentra relacionado con la falta de alternativas profesionales, en comparación con los países de OCDE; aspecto que a la vez se encuentra relacionado a una realidad socioeconómica innegable de alto desempleo, empleos inestables y 
bajos salarios (Rodríguez y Prieto, 2009). Por su parte, GEM (2015) refiere que, en esta región, las empresas creadas por jóvenes en su primera etapa de vida generan empleo únicamente para el propietario.

La proporción de jóvenes de entre 15 a 29 años que poseen un negocio en Latinoamérica es inferior a la proporción de los grupos de edad de entre 30 a 49 años y más de 50, mostrando con esto la falta de impulso y/o oportunidades para emprender hacia los jóvenes de la región, toda vez que comparado con los jóvenes de este mismo grupo de edad de la OCDE la proporción alcanza 6\% vs. $11 \%$ de Latinoamérica, lo cual se debería a la falta de oportunidades para desempeñarse en el ámbito profesional debido a la falta de empleo formal (Ver Figura 6).

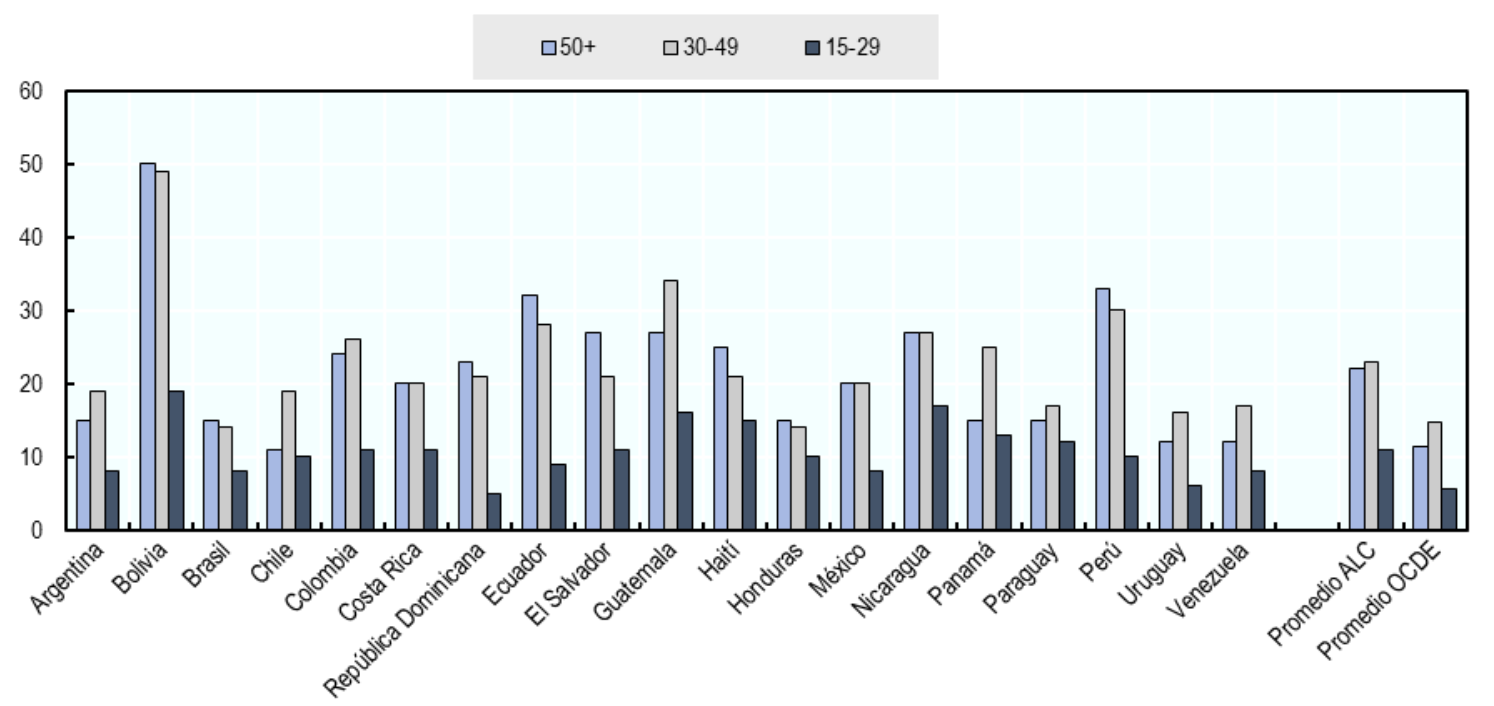

Figura 6. Proporción de la población que posee actualmente un negocio por grupo de edad América Latina y la OCDE.

Fuente: OECD/ECLAC/CAF (2016).

\subsection{El emprendimiento en estudiantes universitarios en Latinoamérica}

En lo que respecta al emprendimiento de estudiantes universitarios Joensuu-Salo, Varamäki y Viljamaa (2015) refieren que las investigaciones no han obtenido resultados contundentes al tratar de establecer la relación entre la educación superior y el emprendimiento. Algunos autores afirman que los estudios reducen la intención de emprendimiento, mientras que otros sugieren que la impulsan, así también, se ha encontrado que los profesionales más jóvenes cuentan con una intención hacia el emprendimiento más fuerte, mientras que otros estudios refieren que son los estudiantes de edad madura los que son impulsados hacia la actividad de emprendimiento. Sin 
embargo, Ahmad y Kumar (2020), encontraron que los individuos que cuentan con estudios de posgrado presentan una intención emprendedora más elevada que los que alcanzaron únicamente estudios profesionales, así también, los que realizaron estudios en áreas empresariales presentan una intención emprendedora más elevada que los de otras áreas.

Un estudio realizado entre estudiantes universitarios de Argentina realizado por CAF (2013) reveló que si bien los estudiantes pertenecientes a diferentes niveles socioeconómicos no difieren en "rasgos psicológicos" (orientación al logro, autonomía, locus de control, innovación y creatividad, autoeficacia, capacidad multitareas) en referencia a su capacidad emprendedora, sí difieren en otros aspectos importantes relacionados con sus perspectivas ocupacionales. Por ejemplo, sus percepciones sobre los retornos de esta actividad son diferentes, puesto que los alumnos con un nivel socioeconómico elevado esperan mayores retornos de la actividad de emprendimiento, aunque este retorno esperado es muy similar a lo que esperan por ejercer su profesión; además, estos alumnos también perciben crecimientos de ingresos más altos a lo largo del ciclo de vida en actividades empresariales, contrario a lo que se observa en el caso de alumnos de menores niveles socioeconómicos.

Este estudio también refiere que en el mediano plazo (hacia los 30 años) los alumnos de niveles socioeconómicos elevados son los que mayormente se inclinan por la actividad de emprendimiento, así como continuar con su preparación universitaria, en comparación con los alumnos de niveles socioeconómicos menores, los cuales manifiestan una menor confianza en sí mismos, en términos de menores expectativas relativas sobre su futuro laboral, suponiendo que tendrán menores salarios en el futuro y que incluso corren el peligro de estar desempleados. Esto podría deberse a que los alumnos de niveles socioeconómicos menores saben que para convertirse en empleadores necesitan contar con recursos de los que carecen, por ejemplo, contar con un capital propio o financiamiento, disponibilidad de una red de contactos apropiada para iniciar un negocio, conocimientos y experiencia.

En la Tabla 1, se presenta una revisión de autores que han estudiado el emprendimiento en estudiantes universitarios en el contexto Latinoamericano, destacando las razones que los impulsan a emprender. Como puede verse, predomina como motivación para emprender el deseo de poner en práctica una idea innovadora, lo que estaría indicando una intención de emprendimiento por oportunidad, y es que es de considerar que el segmento estudiado es el de jóvenes universitarios, los cuales cuentan con una carrera en ciernes, donde el conocimiento adquirido les permite 
formarse una visión de futuro, con la posibilidad de crear empresas rentables. En segundo lugar, el estudiante latinoamericano, es motivado a emprender cuando ha crecido en un entorno empresarial donde los padres o parientes cercanos son o han sido empresarios y cuenta con un modelo a seguir, lo que implica que el contexto familiar y la cultura es importante para los jóvenes de esta región; en tercer lugar, manifiestan como motivo para el emprendimiento, la independencia económica que les brinda el contar con su propia empresa y ser su propio patrón, lo que es señal de status social pues, el emprendedor es visto como una persona exitosa.

\section{Tabla 1}

\section{Estudiantes Universitarios: Motivos para emprender}

\begin{tabular}{|c|c|}
\hline Motivos para emprender & Autores/año \\
\hline $\begin{array}{l}\text { Padres o pariente cercano } \\
\text { emprendedores }\end{array}$ & $\begin{array}{l}\text { Bernales (2010); Alejos y Mireles, 2013; Soria, Zuñiga y Ruiz, 2016; Contreras, } \\
\text { Espinosa, Soria, Portalanza, Jáuregui y Omaña, 2017; Ccama, 2017; Tarapuez, } \\
\text { García y Castellano, 2018. }\end{array}$ \\
\hline $\begin{array}{l}\text { Apoyo de la familia } y \\
\text { amigos }\end{array}$ & $\begin{array}{l}\text { Bernales (2010); Guerrero, Urbano, Ramos, Ruiz-Navarro, Neira y Fernández- } \\
\text { Laviada, 2016; Arévalo, Padilla y Wong 2016; Echeverri, Valencia, Benjumea y } \\
\text { Barrera, 2018. }\end{array}$ \\
\hline Idea innovadora & $\begin{array}{l}\text { Gutiérrez y Amador, 2011; Alejos y Mireles, 2013; Espino y Vásquez, 2015; Durán } \\
\text { y Arias, 2015; Vásquez, 2017; Sánchez, Hernández y Jiménez, 2016; Santos, Pérez } \\
\text { y Haidar, 2017; Jaimes, Jaramillo y Pérez, 2017; Contreras, et al., 2017; Echeverri et } \\
\text { al., 2018; Krauss, Bonomo y Volfovicz, 2018; Pérez-Zavala, Molina, Schmitt y } \\
\text { López, 2019; Amador, García y Díaz, 2020. }\end{array}$ \\
\hline $\begin{array}{l}\text { Independencia } \\
\text { económica }\end{array}$ & $\begin{array}{l}\text { Bernales, 2010; Loli, Del Carpio y Vergara. 2012; Bueckmann, 2014; García, 2015; } \\
\text { Espino y Vásquez, 2015; Arteaga, Mexicano y González, 2016; Tarapuez, 2016; } \\
\text { Vásquez, 2017; Echeverri et al., 2018; Liza, 2018; Amador et al., 2020. }\end{array}$ \\
\hline Evitar la injusticia laboral & Osorio y Londoño, 2015; Echeverri et al., 2018. \\
\hline Realización personal & $\begin{array}{l}\text { Bernales, 2010; Loli et al., 2012; Espino y Vásquez, 2015; Tarapuez, 2016; Vásquez, } \\
\text { 2017, Echeverri et al., 2018, Liza, } 2018 .\end{array}$ \\
\hline $\begin{array}{l}\text { Aprovechar oportunidad } \\
\text { del mercado }\end{array}$ & Echeverri et al., 2018; González, Becerril y Fonseca, 2018. \\
\hline
\end{tabular}

Fuente: elaboración propia con base en los autores citados.

Otras motivaciones que señalaron los estudiantes, son el contar con el apoyo de familia y amigos, sobre todo cuando son escasos los programas de fomento que impulsan el emprendimiento en jóvenes universitarios; evitar la injusticia laboral, que estaría relacionado con los bajos salarios que reciben los jóvenes profesionales; aprovechar una oportunidad del mercado, que tendría relación con los cambios en la sociedad; y por último, aunque no menos importante se encuentra la realización personal, que tiene relación con la necesidad de logro. 
Así como, existen motivaciones, también se presentan barreras para que los jóvenes lleven adelante sus aspiraciones, en este sentido, Worldwide (2014) refiere que en Latinoamérica las PYMES cumplen un papel fundamental en el desarrollo económico y social, más, sin embargo, los jóvenes se enfrentan a una serie de barreras que dificultan su inclusión en esta actividad, carecen de entrenamiento y tutoría para emprender, capital para invertir, canales para ofrecer sus productos o servicios.

En referencia a las necesidades de los estudiantes al momento de emprender, manifestaron la carencia de recursos financieros para iniciar un emprendimiento (Santos et al., 2017; García, 2015), también desataca el hecho de que enfrentan un ambiente poco alentador hacia la actividad de emprendimiento (García, Díaz y Melchor, 2016); el desafió hacia una educación emprendedora (Gutiérrez y Amador, 2011; Sánchez et al., 2016), aunque en referencia a este aspecto otros estudiosos han encontrado que los jóvenes estudiantes sugieren que si reciben en sus instituciones la formación en el espíritu empresarial y que estos programas motivan el emprendimiento (Bueckmann, 2014; Iracheta, Hernández, Cruz y Fernández, 2015; García et al., 2016; Arteaga, et al., 2016; Amador et al., 2020).

Por su parte, Hulsink y Koek (2014) refieren que, aunque los jóvenes carecen de experiencia, capital social, capital financiero y capital humano para emprender no lo consideran como obstáculo y son capaces de iniciar un negocio con poco capital, buscando el apoyo de sus padres, familiares o amigos, captando a sus clientes con creatividad, originalidad y energía, adquiriendo la experiencia y contactos para establecer su empresa. En lo que respecta al capital financiero, Sykes, Elder, Gurbuzer y Principi, (2016) y OECD (2020) refieren que los jóvenes se encuentran excluidos de los servicios financieros, en función a las altas tasas de interés y comisiones que cobran en el sistema financiero, así como por la falta de un ahorro mínimo que pueda servir de garantía de un préstamo y un historial crediticio limitado; en este sentido, en América Latina y el Caribe solo el 37\% de los jóvenes de 15 a 24 años poseen una cuenta en una institución financiera formal, lo cual queda muy rezagado en comparación de los jóvenes que viven en los países de altos ingresos de la OCDE, donde el $84 \%$ de estos jóvenes poseen una cuenta, aunque esto no significa necesariamente que todos sus necesidades financieras se estén cubriendo.

En este tenor el empleo juvenil es tan importante que las Naciones Unidas han lanzado la iniciativa Decent Jobs for Youth bajo la agenda 2030 de desarrollo sostenible, la cual tiene como objetivo lanzar políticas que garanticen empleos decentes para los jóvenes, su desarrollo se basa en ocho 
temáticas: Empleos verdes para jóvenes, habilidades digitales para jóvenes, aprendizajes de calidad, jóvenes en situaciones frágiles, juventud en transición a la economía formal, juventud en el campo economía, emprendimiento juvenil y trabajo por cuenta propia, trabajadores jóvenes en ocupaciones peligrosas (Naciones Unidas, 2020). También, ha desarrollado una estrategia para la juventud que se basa en tres pilares (OIT, 2017): 1) mayor empoderamiento económico de los jóvenes, 2) mayor compromiso cívico y participación de los jóvenes en la toma de decisiones, la vida política y las instituciones públicas, y 3) fortalecimiento de la participación de los jóvenes en la construcción de resiliencia.

Esto debido a que para empoderar económicamente a los jóvenes se requiere fomentar el trabajo decente y abatir el desempleo; así como el apoyo a iniciativas emprendedoras facilitando el acceso a fuentes de financiamiento y capacitación para emprendimiento, puesto que las microempresas ofrecen una salida al problema del desempleo juvenil y deben ser apoyadas (García, 2015; OIT, 2017). El empoderamiento político de los jóvenes, también es necesario a fin de que, a través de la comprensión y asunción de la defensa de sus derechos, articulen sus demandas a través de los canales existentes, empoderándose así para participar en la sociedad civil, la administración pública y la vida política a todo nivel (OIT, 2017). Así también, los jóvenes son agentes para la resiliencia en una comunidad, por lo que es necesario implementar medidas que les garanticen el sustento, fortaleciendo así la cohesión social (OIT, 2017).

\section{MÉTODO}

Se utilizó el método de investigación documental, revisando estudios realizados por organismos internacionales tales como la Organización para la Cooperación y Desarrollo Económico (OCDE), la Organización Internación de Trabajo (OIT; ILO por sus siglas en inglés), la Corporación Andina de Fomento (CAF), entre otras, así como trabajos científicos realizados sobre los temas desempleo juvenil, educación y emprendimiento juvenil con énfasis en emprendimiento universitario.

Se realizó un estudio descriptivo que proporciona los antecedentes sobre el tema, emprendimiento juvenil y universitario, que permitirá la realización de estudios más profundos sobre este tópico. 


\section{DISCUSIÓN}

El problema del desempleo juvenil se encuentra latente en Latinoamérica, ocasionando un desequilibrio económico y social cada vez más sombrío para este sector de la población, el cual por necesidad económica abandona los estudios a temprana edad y se inserta al mercado laboral con gran desventaja, al tener solo la posibilidad de trabajar en el sector informal, creándose así un círculo vicioso que lo estanca en un nivel de pobreza y pobreza extrema del que le es difícil salir.

En la Región Latinoamericana, el empleo se encuentra ligado al nivel de estudios y al nivel socioeconómico de los jóvenes, y contradictoriamente las tasas de desempleo son más elevadas, cuando más elevado es el nivel de estudios y el nivel socioeconómico y son más bajas en la población juvenil de menores estudios y bajo nivel socioeconómico esto se debe principalmente a las altas expectativas que tienen los profesionistas de los niveles socioeconómicos más elevados, acerca de los salarios y prestaciones que podrían obtener del mercado laboral, el cual al encontrarse deprimido y con alta oferta de profesionales, no cubre sus expectativas económicas y prefieren esperar para encontrar mejores oportunidades; mientras que los jóvenes de bajos niveles socioeconómicos se emplean lo más pronto posible aceptando las condiciones que se les ofrece generalmente en el sector informal.

Siendo que la educación puede mejorar las posibilidades de conseguir mejores empleos, es necesario mejorar la cobertura y calidad de la educación, e impulsar los estudios en carreras CTIM (Ciencia, Tecnología, Ingeniería, Matemáticas) con el fin de brindar a los jóvenes conocimientos técnicos y habilidades que se requieren en las organizaciones cambiantes del contexto actual y futuro, donde los cambios tecnológicos marcan la pauta, más aun cuando se conocen los resultados de estudios que refieren por parte de los propios jóvenes acerca de que muy poco de lo aprendido es aplicado en el ámbito laboral, y lo que refieren los empleadores acerca de necesidad que existe de contar con jóvenes capaces de cubrir los perfiles que requieren las empresas.

Aún, cuando el emprendimiento, podría verse como parte de la solución al problema del desempleo en la Región Latinoamericana, son muy pocos aún los jóvenes que se aventuran a emprender, las razones son muchas, entre estas se encuentra la falta de incentivos económicos (capital) y capacitación en habilidades empresariales. Los jóvenes con estudios universitarios, estarían en mayor ventaja para emprender al contar con niveles de estudios más elevados y expresan como mayor motivación para ello la puesta en marcha de una idea innovadora lo cual traería como 
consecuencia la creación de una empresa competitiva y rentable, puesto que la innovación marca la pauta en los mercados hoy en día.

A pesar, de la importancia del emprendimiento juvenil y la implementación de programas para impulsarlo son muchas las barreras que aún se encuentran para fomentar esta actividad y así convertirse en una solución viable al problema del desempleo en Latinoamérica.

\section{CONCLUSIONES}

Las pocas oportunidades de empleo que existen para los jóvenes Latinoamericanos, se ve acentuado por las crisis económicas globales, pues son los jóvenes principalmente los que trabajan en empleos, temporales, informales y precarios, los cuales son los primeros en desaparecer lanzándolos así a una situación de desempleo, que acentúa aún más los círculos de pobreza en esta región.

Aunque el emprendimiento podría ser la solución al problema del desempleo juvenil, los estudios antecedentes señalan que en esta región predomina el emprendimiento de subsistencia, originado por jóvenes de estratos socioeconómicos y niveles de estudios bajos, lo que se convierte en un círculo vicioso de baja productividad. Los jóvenes con estudios profesionales, estarían contando con mejores herramientas para emprender, la principal es el conocimiento que permite generar bienes y servicios innovadores capaces de competir en los mercados cada vez más saturados, pero donde el cliente tiene la posibilidad de elegir los que realmente satisfagan sus necesidades.

Es necesario entonces, que las políticas en torno a la promoción del emprendimiento, en los países de la región, consideren el segmento juvenil y universitario, con el fin de establecer programas de apoyo que realmente los beneficio, los cuales deberían de ir desde la capacitación para la obtención de habilidades empresariales, hasta la facilidad para acceder a fuentes de financiamiento acordes a sus necesidades y realidad social.

\section{REFERENCIAS}

(1) Acosta, J., Zárate, R. e Ibarra, A. (2014). Caracterización del emprendedor Latinoamericano a partir del Modelo Global Enterpreneurship Monitor - GEM”. Revista Económicas CUC, 35(1), 135-155. Recuperado de https://revistascientificas.cuc.edu.co/economicascuc/article/view/212 
(2) Ahmad, Z. y Kumar, S. (2020). Does entrepreneurship education influence entrepreneurial intention among students in HEI's? The role of age, gender and degree background, Journal of International Education in Business, 13(1), 55-72. doi: 10.1108/JIEB-02-2019-0009

(3) Alejos, A. y Mireles, L. (2013). Espíritu emprendedor en estudiantes de educación superior. Pistas Educativas, 106, 98-104.

(4) Amador, M., García, Y. y Díaz, J. (2020). Comparación del perfil emprendedor en estudiantes del área empresarial e industrial México-Perú. Revista Iberoamericana para la Investigación y el desarrollo Educativo, 10(20), 1-22. doi: 10.23913/ride.v10i20.598

(5) Appie, S., Eresia, C. y Gervase, C. (2014). Challenges and Prospects of Youth Entrepreneurship Development in a Designated Community in the Western Cape, South Africa. Journal of Economics and Behavioral Studies, 6(1), 10-20.

(6) Arévalo, D., Padilla, C. y Wong, N. (2016). Intención de emprendimiento en los estudiantes: Un estudio en la Universidad Católica de Santiago de Guayaquil. Alternativas, 17(1), 5-15. doi: 10.23878/alternativas.v17i1.106

(7) Arteaga, A., Mexicano, M. y González, R. (2016). Intención emprendedora en estudiantes de la licenciatura de administración en una institución de educación superior. XXI Congreso Internacional de Contaduría, Administración e Informática. México: FCA-UNAM.

(8) Bernales, M. (2010). Intención Emprendedora y Factor de Migración en Estudiantes Universitarios del Perú (Tesis doctoral). Universidad de Salamanca, España.

(9) Bueckmann, R. (2014). Análisis de las Intenciones Empresariales de los Estudiantes Universitarios Mexicanos: Un enfoque Basado en variables personales y del programa educativo (Tesis doctoral). Universidad de Cantabria, España.

(10) CAF. (2013). Emprendimientos en América Latina: Desde la subsistencia hacia la transformación productiva. Bogotá, Colombia: Corporación Andina de Fomento.

(11) Ccama, S. (2017). Análisis del Emprendimiento Universitario y la Intención Emprendedora en los Estudiantes de Administración de la Universidad Nacional Del Altiplano - 2016 (Tesis de pregrado). Universidad Nacional del Altiplano, Perú.

(12) Contreras, F., Espinosa, J. C., Soria, K., Portalanza, A., Jáuregui, K. y Omaña, J. A. (2017). Exploring entrepreneurial intentions in Latin American university students. International Journal of Psychological Research, 10(2), 46-59. doi: 10.21500/20112084.2794

(13) Durán, E. y Arias, D. (2015). Intención emprendedora en estudiantes universitarios: integración de factores cognitivos y socio-personales. Revista Colombiana de Ciencias Sociales, 6(2), 320-340. doi: $10.21501 / 22161201.1528$

(14) Echeverri, L., Valencia, A., Benjumea, M. y Barrera, A. (2018). Factores que inciden en la intención emprendedora del universitario: Un análisis cualitativo. Revista Electrónica Educare, 22(2), 21-19. doi: $10.15359 /$ ree.22-2.10 
(15) Espino, A. y Vázquez, S. (2015). Intención emprendedora en estudiantes universitarios de Ingeniería Industrial Administrativa de la Universidad Católica Santa María la Antigua (USMA). Invest. pens. crit., 3(3), 3-26.

(16) García, B., Díaz, M. y Melchor, L. (2016). Estudiantes de la Carrera de Administración de Empresas de la Universidad Autónoma de Aguascalientes y su Intención Emprendedora. Red Internacional de Investigadores en Competitividad, IX, (1), 1695-1710.

(17) García, V. D. (2015). Emprendimiento Empresarial Juvenil: Una evaluación con jóvenes estudiantes de universidad. Revista Latinoamericana de Ciencias Sociales, Niñez y Juventud, 13(2), 1221-1236.

(18) GEM. (2015). Future Potential. A GEM perspective on youth entrepreneurship 2015. Massachusetts: Babson College.

(19) González, G., Becerril, M. y Fonseca, A. (2018). El Engagement como Factor de Formación y Desarrollo de la Cultura Emprendedora en Estudiantes Universitarios. IE Revista de Investigación Educativa de la REDIECH, 9(17), 103-118.

(20) Guerrero, M., Urbano, D., Ramos, A., Ruiz-Navarro, Neira, J. y Fernández-Laviada, A. (2016). Perfil Emprendedor de estudiantes Universitarios. Madrid: Crue Universidades Españolas-RedEmprendiaCISE.

(21) Gutiérrez, A. y Amador, M. (2011). El potencial emprendedor en los estudiantes de la carrera de contabilidad de las Universidades San Marcos de Perú y Guadalajara de México - Centro Universitario de los Altos - un análisis comparativo. Revista de la Facultad de Ciencias Contables, 19(36), 63-82.

(22) Hulsink, W. y Koek, D. (2014). The young, the fast and the furious: a study about the triggers and impediments of youth entrepreneurship. Int. J. Entrepreneurship and Innovation Management, $8(2 / 3), 182-209$.

(23) ILO. (2017). Global Employment Trends for Youth 2017: Paths to a better working future. Geneva: International Labour Office.

(24) ILO. (2018). Labour Overview 2018. Latin America and the Caribbean. Lima: ILO/ Regional Office for Latin America and the Caribbean.

(25) Iracheta, J., Hernández, R., Cruz, M. y Fernández, A. (2015). Estudio de la Intención Emprendedora en los Estudiantes Universitarios. Universidad de Extremadura, 1, 1-13.

(26) Jaimes, F., Jaramillo, M. y Pérez, M. (2017). Factores que inciden en la intención emprendedora de estudiantes del Centro Universitario Temascaltepec. Revista Venezolana de Gerencia, 22(78), 210231. doi: 10.37960/revista.v22i78.22875

(27) Joensuu-Salo, S., Varamäki, E. \& Viljamaa, A. (2015). Beyond intentions - what makes a student start a firm? Education + Training, 57(8/9), 853-873. doi: 10.1108/ET-11-2014-0142

(28) Krauss, C., Bonomo, A. \& Volfovicz, R. (2018). Modelo predictivo de la intención emprendedora universitaria en Latinoamérica. Journal of Technology Management \& Innovation, 13(4), 84-93. doi: 10.4067/S0718-27242018000400084 
(29) Liza, I. (2018). Relación entre Intención Emprendedora, Norma Subjetiva, Autoeficacia Emprendedora y Personalidad Proactiva en un grupo de estudiantes de una universidad privada de Lima (Tesis de pregrado). Universidad Peruana de Ciencias Aplicadas (UPC), Lima, Perú.

(30) Loli, A., Del Carpio, J. \& Vergara, A. (2012). Actitudes de Emprendimiento, Necesidad de Logro y la Intención de Desarrollar un Negocio en Estudiantes de Universidades Públicas de Lima Metropolitana. Revista IIPS, 15(1), 61-81. doi: 10.15381/rinvp.v15i1.3662

(31) Naciones Unidas (2020). Decent Jobs for Youth. Youth entreprenuership and Self Employment. Santiago: Naciones Unidas.

(32) OECD. (2019). SME and Entrepreneurship Outlook, 2019. Paris: OECD Publishing.

(33) OECD. (2020). Supporting Youth Entrepreneurship in a Review of Policies and Programmes Spain. Paris: OECD Publishing.

(34) OECD/ECLAC/CAF. (2016). Latin American Economic Outlook 2017: Youth, Skills and Entrepreneurship. Paris: OECD Publishing.

(35) OIT. (2017). El futuro del trabajo que queremos. La voz de los jóvenes y diferentes miradas desde América Latina y el Caribe. Lima: OIT, Oficina Regional para América Latina y el Caribe, Informes técnicos, 2017/7.

(36) OIT. (2019). OIT Panorama Laboral 2019. Lima: OIT/Oficina Regional para América Latina y el Caribe.

(37) Osorio, F. y Londoño, J. (2015). Intención emprendedora de estudiantes de educación media: extendiendo la teoría de comportamiento planificado mediante el efecto exposición. Cuadernos de administración, 28(51), 103-131. doi: 10.11144/Javeriana.cao28-51.ieee

(38) Pérez-Zavala, M., Molina, R., Schmitt, C. y López, A. (2019). Emprendimiento en dos universidades públicas: Universidad de Guanajuato, México y Université de Lorraine, France. Projectics / Proyéctica / Projectique, 23(2), 99-116.

(39) Rodríguez, C. y Prieto, F. (2009). La sensibilidad al emprendimiento en los estudiantes universitarios. Estudio comparativo Colombia-Francia. INNOVAR, Revista de Ciencias Administrativas y Sociales, 19, 73-89.

(40) Sánchez, S., Hernández, C. A. y Jiménez, M. (2016). Análisis de la percepción sobre iniciativa empresarial y el espíritu emprendedor en estudiantes de un tecnológico federal. Acta Universitaria, 26(6), 70-82. Doi: 10.15174/au.2016.1016

(41) Santos, S. Pérez, A. y Haidar, E. (2017). Motivación al logro, innovación y control interno como factores cognitivos condicionales de la intención empresarial en estudiantes universitarios. XXII Congreso Internacional de Contaduría, Administración e Informática. México: FCA-UNAM, octubre.

(42) Soria, K., Zuñiga, S. y Ruiz, S. (2016). Educación e Intención Emprendedora en Estudiantes Universitarios: Un Caso de Estudio. Formación Universitaria, 9(1), 25-34. doi: 10.4067/S071850062016000100004 
(43) Sykes, J., Elder, S., Gurbuzer, Y. y Principi, M. (2016). Exploring the linkages between youth financial inclusion and job creation: evidence from the ILO school to work transition surveys. Geneve: ILO, International Labour Office.

(44) Tarapuez, E. (2016). Las dimensiones culturales de Geert Hofstede y la intención emprendedora en estudiantes universitarios del departamento del Quindío (Colombia). Pensamiento \& Gestión, 41, 6090.

(45) Tarapuez, E., García, M. y Castellano, N. (2018). Aspectos socioeconómicos e intención emprendedora en estudiantes universitarios del Quindío (Colombia). Innovar, 28(67), 123-135. doi: 10.15446/innovar.v28n67.68618

(46) Vásquez, J. (2017). Factores que inciden en la intención de emprendimiento de universitarios adultos con experiencia laboral (Tesis doctoral). Universidad San Ignacio De Loyola, Perú.

(47) Worldwide, JA. (2014). Quoting Generation Jobless: The Challenge of Global Youth Unemployment with attribution is encouraged. Boston, USA: JA Worldwide.

Cómo citar este artículo: Saavedra García, M. (2020). El desempleo juvenil en Latinoamérica y el emprendimiento de estudiantes universitarios. Tendencias, 21(2), 283-305. https://doi.org/10.22267/rtend.202102.151 